\title{
BINARY AND TERNARY TRANSFORMATIONS OF SEQUENCES
}

\author{
by D. BORWEIN and A. V. BOYD
}

(Received 29th June 1958; revised MS. received 26th September 1958)

\section{Introduction}

Agnew (1) has defined a binary transformation $T(\alpha)$, with $\alpha$ real, as one which takes the sequence $\left\{s_{i}\right\}, i=0,1, \ldots$, into the sequence $\left\{s_{i}(1, \alpha)\right\}$ where

$$
s_{i}(1, \alpha)=\left\{\begin{array}{l}
\alpha s_{0} \text { for } i=0, \\
\alpha s_{i}+(1-\alpha) s_{i-1}
\end{array} \text { for } i=1,2, \ldots .\right.
$$

An $r$-fold application of $T(\alpha)$ yields the transformation $T^{r}(\alpha)$ which takes $\left\{s_{i}\right\}$ into $\left\{s_{i}(r, \alpha)\right\}$ where, in general, if $s_{n}(0, \alpha)=s_{n}$ and $s_{n}(r, \alpha)=0$ for negative integral $n$ then, for all $n$ and $l \geqslant 0$,

$$
s_{n}(l+1, \alpha)=\alpha s_{n}(l, \alpha)+(1-\alpha) s_{n-1}(l, \alpha) .
$$

It easily follows by induction that

$$
s_{n}(l+r, \alpha)=\sum_{k=0}^{r}\left(\begin{array}{l}
\dot{r} \\
k
\end{array}\right)(1-\alpha)^{r-k} \alpha^{k} s_{n-r+k}(l, \alpha),
$$

with the convention that $0^{0}=1$.

Putting $l=0, q=1 / \alpha-1,(\alpha \neq 0)$, we obtain

$$
s_{n}(r, \alpha)=(q+1)^{-r} \sum_{k=n-r}^{n}\left(\begin{array}{c}
r \\
n-k
\end{array}\right) q^{n-k} s_{k}
$$

and

$$
s_{n}(n, \alpha)=(q+1)^{-n} \sum_{k=0}^{n}\left(\begin{array}{l}
n \\
k
\end{array}\right) q^{n-k_{s}} s_{k}
$$

If $s_{n}(r, \alpha)$ tends to a finite limit $s$ as $n$ tends to infinity then $\left\{s_{i}\right\}$ is said to be summable $T^{r}(\alpha)$ to $s$. If $s_{n}(n, \alpha)$ tends to a finite limit $s$ as $n$ tends to infinity then $\left\{s_{i}\right\}$ may be said to be summable $T^{\infty}(\alpha)$ to $s$. From (iii) and Hardy (2), equation (8.3.4), it follows that summability $T^{\infty}(\alpha)$ is equivalent to Euler summability $(E, q)$. It should also be noted that summability $T^{0}(\alpha)$ is equivalent to convergence.

We shall use the notation $P \Rightarrow Q$ to mean that any sequence summable $(P)$ to $s$ is necessarily summable $(Q)$ to $s$, and $P \Leftrightarrow Q$ to mean that both $P \Rightarrow Q$ and $Q \Rightarrow P$.

\section{Relations between $T^{r}(\alpha)$ and $T^{\infty}(\alpha)$}

Knopp (5) has shown that for $0<\alpha \leqslant 1$ convergence to $s$ implies summability $(E, 1 / \alpha-1)$ to $s$, i.e. that $T^{0}(\alpha) \Rightarrow T^{\infty}(\alpha)$; and from a general result on compounded matrices Agnew (1) has deduced that $T^{r}(\alpha) \Rightarrow T^{\infty}(\alpha)$ for $r \geqslant 0,0<\alpha<1$. 
The case $\alpha=\frac{1}{2}$ of this result was familiar to Hutton (3) who first considered the $T^{r}\left(\frac{1}{2}\right)$ process early in the nineteenth century without giving rigorous proofs. The following proof is more direct than Agnew's.

Theorem. For $r \geqslant 0$,

(i) $T^{r}(\alpha) \Rightarrow T^{r+1}(\alpha)$ for any $\alpha$;

(ii) $T^{r}(\alpha) \Rightarrow T^{\infty}(\alpha)$ if and only if $0<\alpha \leqslant 1$.

Proof. (i) is trivial.

(ii) Sufficiency. Let $q=1 / \alpha-1 \geqslant 0$ and suppose that $\left\{s_{n}\right\}$ is summable $T^{r}(\alpha)$ to $s$. Applying the $(E, q)$ process, which is known (see e.g. (2), p. 179) to be regular for $q \geqslant 0$, to the sequence $s_{r}(r, \alpha), s_{r+1}(r, \alpha), s_{r+2}(r, \alpha), \ldots$ which converges to $s$, we get that

$$
(q+1)^{-n} \sum_{k=0}^{n}\left(\begin{array}{l}
n \\
k
\end{array}\right) q^{n-k_{s}} s_{k+r}(r, \alpha) \rightarrow s \text { as } n \rightarrow \infty .
$$

In virtue of identity (i) with $r, l$ and $n$ replaced by $n, r$ and $n+r$ respectively, it follows that $\left\{s_{n}\right\}$ is summable $T^{\infty}(\alpha)$ to $s$.

Necessity. If $s_{n}=(1-2 / \alpha)^{n}$ then the $T^{\infty}(\alpha)$ transform of $\left\{s_{n}\right\}$ is $\left\{(-1)^{n}\right\}$; and for $\alpha>1,\left\{s_{n}\right\}$ is summable $T^{0}(\alpha)$ and so summable $T^{r}(\alpha)$, but is not summable $T^{\infty}(\alpha)$.

If $\left\{s_{n}\right\}$ is the sequence $1,0,0, \ldots$ then its $T^{\infty}(\alpha)$ transform is $\left\{(1-\alpha)^{n}\right\}$. For every $\alpha,\left\{s_{n}\right\}$ is summable $T^{0}(\alpha)$, and so summable $T^{r}(\alpha)$, to 0 ; but the sequence is summable $T^{\infty}(0)$ to 1 and is not summable $T^{\infty}(\alpha)$ for any $\alpha<0$.

The condition $0<\alpha \leqslant 1$ is therefore necessary.

\section{Nörlund means, etc.}

The following results will be used later :

Kubota's theorem. (6). If $a_{0}, a_{1}, \ldots, a_{k}\left(a_{k} \neq 0\right)$ are fixed real or complex numbers then, in order that $x_{n}$ should tend to $l /\left(a_{0}+a_{1}+\ldots+a_{k}\right)$ whenever $a_{0} x_{n-k}+a_{1} x_{n-k+1}+\ldots+a_{k} x_{n}$ tends to $l$, it is necessary and sufficient that all roots of the equation $a_{0}+a_{1} x+\ldots+a_{k} x^{k}=0$ should lie within the unit circle.

Nörlund means. Suppose that $p_{0} \neq 0, P_{n}=p_{0}+p_{1}+\ldots+p_{n}$ where $p_{n}$ is real, and that $P_{n} \neq 0$ for $n \geqslant M$.

For $\quad n \leqslant M$ let $t_{n}=\sum_{k=0}^{n} p_{n-k} s_{k} / P_{M}$

and for $n \geqslant M$ let $t_{n}=\sum_{k=0}^{n} p_{n-k} s_{k} / P_{n}$.

We shall say that sequence $\left\{s_{n}\right\}$ is summable by the Nörlund method $\left(N, p_{n}\right)$ to $s$ if $t_{n}$ tends to $s$ as $n$ tends to infinity. In (2), Hardy imposes the further condition $p_{n} \geqslant 0$ (and takes $M=0$ ), but this is too restrictive for our purposes. 
It follows from formula (ii) that for $\alpha \neq 0$ the $T^{r}(\alpha)$ transformation is a Nörlund transformation with

and

$$
\begin{aligned}
& M=r, p_{n}=\left\{\begin{array}{l}
\left(\begin{array}{l}
r \\
n
\end{array}\right) p^{n}, p=1 / \alpha-1, \text { for } 0 \leqslant n \leqslant r, \\
0 \text { for } n>r,
\end{array}\right. \\
& P_{n}=(1+p)^{r} \text { if } n \geqslant r,
\end{aligned}
$$

It is also known (2, p. 109) that the Cesàro mean $(C, r)$ with $r \geqslant 0$ can be expressed as a Nörlund mean $\left(N, p_{n}\right)$ with

and

$$
M=0, p_{n}=\left(\begin{array}{c}
n+r-1 \\
r-1
\end{array}\right) \sim \frac{n^{r-1}}{\Gamma(r)} \text { if } r>0,
$$

For $r \geqslant 0, \sum_{n=0}^{\infty} p_{n} x^{n}=(1-x)^{-r}$ and $P_{n} \sim \frac{n^{r}}{\Gamma(r+1)}$.

The following simple extensions of Hardy's theorems 16, 17, 19 and 21 can be established by using the methods of his proofs and (in the case of theorem 17) a result due to Jurkat and Peyerimhoff (4, lemma 1).

Theorem 16. The Nörlund method $\left(N, p_{n}\right)$ is regular, i.e. the convergence of a sequence to a finite limit implies its summability $\left(N, p_{n}\right)$ to the same limit, if and only if there is a constant $H$ independent of $n$ such that

$$
\sum_{r=0}^{n}\left|p_{r}\right|<H\left|P_{n}\right| \text { for } n \geqslant M
$$

and $p_{n} / P_{n} \rightarrow 0$ as $n \rightarrow \infty$.

Theorem 17. Any two regular Nörlund methods $\left(N, p_{n}\right),\left(N, q_{n}\right)$ are consistent; i.e. if a sequence is summable $\left(N, p_{n}\right)$ to $s$ and $\left(N, q_{n}\right)$ to $t$ then $s=t$.

Theorem 19. If $\left(N, p_{n}\right)$ and $\left(N, q_{n}\right)$ are regular and $p(x)=\Sigma p_{n} x^{n}$, $q(x)=\Sigma q_{n} x^{n}, q(x) / p(x)=\Sigma k_{n} x^{n}$, then in order that summability $\left(N, p_{n}\right)$ of a sequence should imply its summability $\left(N, q_{n}\right)$ it is necessary and sufficient that

$$
\sum_{r=0}^{n}\left|k_{n-r} P_{r}\right|<H\left|Q_{n}\right| \text { for } n \geqslant M
$$

where $H$ is independent of $n$, and that $k_{n} / Q_{n} \rightarrow 0$.

Theorem 21. A necessary condition that two regular Nörlund methods $\left(N, p_{n}\right)$ and $\left(N, q_{n}\right)$ be equivalent is that $\Sigma\left|k_{n}\right|$ and $\Sigma\left|l_{n}\right|$ be finite, where $\Sigma l_{n} x^{n}=p(x) / q(x)$.

Corollary. Regular Nörlund methods $\left(N, p_{n}\right)$ and $\left(N, q_{n}\right)$ cannot be equivalent if $p(x)$ and $q(x)$ are rational and one of them has a zero, inside or on the unit circle, which is not a zero of the other. 
In the case of the $T^{r}(\alpha)$ process $p(x)$ has a zero at $x=-1 / p=\alpha /(\alpha-1)$ if $\alpha \neq 0$; while $T^{r}(0) \Leftrightarrow T^{r}(1)$, and for $T^{r}(1) p(x)$ has no zero. It follows from the corollary that if $\alpha \leqslant \frac{1}{2}, \beta \leqslant \frac{1}{2}$ and $\alpha \neq \beta$ then $T^{r}(\alpha)$ and $T^{s}(\beta)$ cannot be equivalent for any $\alpha, \beta, r, s$.

\section{Relation of $T^{r}(\alpha)$ to the Cesàro and Abel processes}

If $\left(N, p_{n}\right)$ is taken as the $(C, s)$ process with $s>0$, and $\left(N, q_{n}\right)$ as $T^{r}(\alpha)$, then

$$
k(x)=(1+p x)^{r}(1-x)^{s}, \quad\left|k_{0}\right| P_{n} \sim n^{s} / \Gamma(s+1)
$$

and $Q_{n}=\alpha^{-r}$ for $n \geqslant r$.

By theorem 19 it follows that, for $s>0$, summability $(C, s)$ cannot imply summability $T^{r}(\alpha)$. In the reverse direction we have the following results :

$\alpha>\frac{1}{2}$. By Kubota's theorem a sequence which is $T(\alpha)$ summable to $s$ converges to $s$ if and only if $|(\alpha-1) / \alpha|<1$, i.e. if and only if $\alpha>\frac{1}{2}$. Since the $T^{r}(\alpha)$ transform is the $T(\alpha)$ transform of the $T^{r-1}(\alpha)$ transform it follows that $(C, 0) \Leftrightarrow T^{r}(\alpha)$ for $\alpha>\frac{1}{2}$.

$\alpha=\frac{1}{2}$. Taking $\left(N, p_{n}\right)$ and $\left(N, q_{n}\right)$ as $T^{r}\left(\frac{1}{2}\right)$ and $(C, r)$ respectively we get $k(x)=\left(1-x^{2}\right)^{-r}$ so that

$$
k_{n}=\left\{\begin{array}{cc}
\left(\begin{array}{c}
2+r-1 \\
r-1
\end{array}\right) & \text { when } n \text { is even } \\
0 & \text { when } n \text { is odd }
\end{array}\right.
$$

For large $n, k_{n}=O\left(n^{r-1}\right), k_{n} / Q_{n}=O(1 / n)=o(1)$, and

$\left\{\left|k_{0}\right| P_{n}+\ldots+\left|k_{n}\right| P_{0}\right\} / Q_{n}=O\left(n^{r} / n^{r}\right)=O(1)$, so that $T^{r}\left(\frac{1}{2}\right) \Rightarrow(C, r)$.

The result is "best possible" in the sense that, for any integer $r$ there is a sequence which is summable $T^{r}\left(\frac{1}{2}\right)$ but which is not summable $(C, r-\delta)$ for any $\delta>0$. This is shown by considering the example $s_{n}=(-1)^{n} n^{r} / \log n$, the case $r=1$ of which is due to Silverman and Szasz (7). Since $s_{n} \neq o\left(n^{r-\delta}\right), \delta>0$, the sequence $\left\{s_{n}\right\}$ is not summable $(C, r-\delta)$.

If, however, $s_{n}=(-1)^{n} f(n)$ where $f(n)$ is a polynomial of degree $m$ then $s_{n}\left(1, \frac{1}{2}\right)=(-1)^{n} \frac{1}{2}\{f(n)-f(n-1)\}=(-1)^{n} g(n)$, where $g(n)$ is a polynomial of degree $m-1$. Hence

$$
s_{n}\left(r, \frac{1}{2}\right)=O\left(n^{m-r}\right) .
$$

Putting $f(n)=n^{r+s}$ (s a non-negative integer) gives

$$
\sum_{k=0}^{r}(-1)^{k}\left(\begin{array}{l}
r \\
k
\end{array}\right)(n-k)^{r+s}=O\left(n^{s}\right) ;
$$

from which it easily follows, on using the identity $k=n-(n-k)$, that

$$
\sum_{k=0}^{r}(-1)^{k}\left(\begin{array}{l}
r \\
k
\end{array}\right) k^{s}(n-k)^{r}=O\left(n^{s}\right)
$$


Further, for $n-2 \geqslant r \geqslant 1, r \geqslant k \geqslant 0$, we have

$$
\begin{aligned}
\frac{\log n}{\log (n-k)} & =\left\{1+\frac{\log (1-k / n)}{\log n}\right\}^{-1} \\
& =\sum_{r=0}^{r}(-1)^{s}\left\{\frac{\log (1-k / n)}{\log n}\right\}^{s}+O\left\{(n \log n)^{-r}\right\} \\
& =1+A_{1} \frac{k}{n}+A_{2}\left(\frac{k}{n}\right)^{2}+\ldots+A_{r}\left(\frac{k}{n}\right)^{r}+O\left(n^{-r}\right)
\end{aligned}
$$

where the $A$ 's are bounded functions of $n$ independent of $k$. It follows that if

$$
I_{n}=(-1)^{n} 2^{-r} \sum_{k=0}^{r}(-1)^{k}\left(\begin{array}{l}
r \\
k
\end{array}\right)(n-k)^{r} / \log (n-k)
$$

then $I_{n} \log n=O(1)$, so that $I_{n} \rightarrow 0$. But $I_{n}$ is $s_{n}\left(r, \frac{1}{2}\right)$ for the sequence $\left\{(-1)^{n} n^{r} / \log n\right\}$; hence this sequence is summable $T^{r}\left(\frac{1}{2}\right)$ to 0 .

$\alpha<\frac{1}{2}$. If $\alpha=0$ then summability $T^{r}(\alpha)$ is trivially equivalent to convergence. Otherwise consider, as does Agnew (1), the sequence $\left\{s_{n}\right\}$ where $s_{n}=(1-1 / \alpha)^{n}$. It is summable $T(\alpha)$ to 0 and so is also summable $T^{r}(\alpha)$ to 0 , but $\Sigma s_{n} z^{n}$ has radius of convergence $|\alpha /(\alpha-1)|<1$ so that $\left\{s_{n}\right\}$ is not Abel summable. Hence for $\alpha<\frac{1}{2}, \alpha \neq 0$, summability $T^{r}(\alpha)$ does not imply Abel summability.

\section{Ternary transformations}

We may define $T(\alpha, \beta)$ to be the ternary transformation which takes $\left\{s_{n}\right\}$ into the sequence $\left\{s_{n}^{\prime}\right\}$ where

$$
\begin{aligned}
& s_{0}^{\prime}=\alpha s_{0}, s_{1}^{\prime}=\alpha s_{1}+\beta s_{0} \text { and } \\
& s_{n}^{\prime}=\alpha s_{n}+\beta s_{n-1}+(1-\alpha-\beta) s_{n-2}(n=2,3, \ldots) .
\end{aligned}
$$

It follows immediately that $T(\alpha, 1-\alpha)$ is equivalent to $T(\alpha)$, and that the $T(\alpha, \beta)$ transformation is a Nörlund transformation $\left(N, p_{n}\right)$ with $M=2, p_{0}=\alpha$, $p_{1}=\beta, p_{2}=1-\alpha-\beta, p_{n}=0(n \geqslant 3), P_{n}=1(n \geqslant 2), p(x)=\alpha+\beta x+(1-\alpha-\beta) x^{2}$.

\section{Relation of $T(\alpha, \beta)$ to the $(C, 0)$ and Abel processes}

Let $f(x)=\alpha x^{2}+\beta x+1-\alpha-\beta$, and divide the $(\alpha, \beta)$ plane into three disjoint sets as follows. Let $S_{1}, S_{2}, S_{2}$ be respectively the sets of points $(\alpha, \beta)$ for which

(1) $f(x)$ has no zeros in the region $|x| \geqslant 1$,

(2) $f(x)$ has at least one zero in the region $|x|>1$,

(3) $f(x)$ has two zeros, one lying on the circle $|x|=1$ and the other in the region $|x| \leqslant 1$.

(a) It is trivially evident that $T(0,0) \Leftrightarrow(C, 0)$. Hence, by Kubota's theorem, $T(\alpha, \beta) \Leftrightarrow(C, 0)$ if and only if $(\alpha, \beta) \in S_{1}$.

(b) If $(\alpha, \beta) \epsilon S_{2}$, there is a number $s$ such that $|s|>1$ and $f(s)=0$. Hence $\Sigma s^{n} z^{n}$ has radius of convergence $|1 / s|<1$, and so the sequence $\left\{s^{n}\right\}$ is not Abel summable. On the other hand if $s_{n}=s^{n}$ then $s_{n}^{\prime}=s^{n-2} f(s)=0$ so that $\left\{s^{n}\right\}$ is summable $T(\alpha, \beta)$ to 0 . Thus summability $T(\alpha, \beta)$ does not imply summability by Abel's method for $(\alpha, \beta) \in S_{2}$. 
Before investigating the behaviour of $T(\alpha, \beta)$ for $(\alpha, \beta) \epsilon S_{3}$, we delimit the sets $S_{1}, S_{2}$ and $S_{3}$. Since $S_{2}$ is the complement of $S_{1} \cup S_{3}$ it is sufficient to consider only $S_{1}$ and $S_{3}$.

The set $S_{1}$. We show that $S_{1}$ consists of the point $(0,0)$, the part $\beta>\frac{1}{2}$ of the line $\alpha=0$ and the region $2 \alpha+\beta>1, \beta<\frac{1}{2}$.

It is easily seen that $(0, \beta) \epsilon S_{1}$ if and only if $\beta=0$ or $\beta>\frac{1}{2}$. It remains to prove that when $\alpha \neq 0,(\alpha, \beta) \epsilon S_{1}$ if and only if $2 \alpha+\beta>1, \beta<\frac{1}{2}$.

(i) Suppose that both zeros $x_{1}, x_{2}$ of $f(x)$ lie in $|x|<1$. Since $f(-1) \neq 0$ and $f(1)=1, f(-1)=1-2 \beta$ must be positive, for otherwise $f(x)$ would have one real zero in the range $-1<x<1$ and another outside this range. Hence $\beta<\frac{1}{2}$.

Further, $-1<x_{1} x_{2}=-1+(1-\beta) / \alpha<1$, so that $0<(1-\beta) / \alpha<2$. Since $\beta<\frac{1}{2}, \alpha$ must be positive and so $2 \alpha+\beta>1$.

(ii) Suppose $2 \alpha+\beta>1, \beta<\frac{1}{2}$. Then $\alpha>0$ and, as above, $-1<x_{1} x_{2}<1$. Hence, if the zeros of $f(x)$ are not real, both must lie in $|x|<1$. If both zeros are real, one must lie in the range $-1<x<1$ and, since $f(-1)>0, f(1)>0$, so must the other.

The set $S_{\mathbf{3}}$.

(i) $(\alpha, \beta) \in S_{3}$ and $f(x)$ has non-real zeros $x_{1}$ and $x_{2}$ if and only if

$$
x_{1} x_{2}=1=-1+(1-\beta) / \alpha, 4 \alpha>(\beta+2 \alpha)^{2},
$$

which is equivalent to $2 \alpha+\beta=1, \alpha>\frac{1}{4}$.

(ii) Since $f(1)=1,(\alpha, \beta) \in S_{3}$ and $f(x)$ has real zeros if and only if

$$
f(-1)=1-2 \beta=0,|(1-\beta) / \alpha-1| \leqslant 1,
$$

which is equivalent to $\beta=\frac{1}{2}, \alpha \geqslant \frac{1}{4}$.

Hence $S_{3}$ consists of the part $\alpha>\frac{1}{4}$ of the line $2 \alpha+\beta=1$ and the part $\alpha \geqslant \frac{1}{4}$ of the line $\beta=\frac{1}{2}$.

7. Relation of $T(\alpha, \beta)$ to the Cesàro process in $S_{3}$

(i) The segment $\alpha \geqslant \frac{1}{4}$ of the line $\beta=\frac{1}{2}$.

Here

$$
\alpha+\beta x+(1-\alpha-\beta) x^{2}=\alpha(1+x)\left(1+\frac{1-2 \alpha}{2 \alpha} x\right) .
$$

In theorem 19 take $\left(N, p_{n}\right)$ to be the $T(\alpha, \beta)$ process and $\left(N, q_{n}\right)$ the Cesàro $(C, s)$ process. Then

$$
\begin{aligned}
k(x) & =1 /\left\{\alpha(1-x)^{s-1}\left(1-x^{2}\right)\left(1-\frac{2 \alpha-1}{2 \alpha} x\right)\right\} \\
& =\frac{1}{\alpha}\{1+(s-1) x+\ldots\}\left(1+x^{2}+x^{4}+\ldots\right)\left\{1+\frac{2 \alpha-1}{2 \alpha} x+\left(\frac{2 \alpha-1}{2 \alpha} x\right)^{2}+\ldots\right\} .
\end{aligned}
$$

If $s=1$, then

$$
k_{n}=\frac{1}{\alpha}\left\{\left(\frac{2 \alpha-1}{2 \alpha}\right)^{n}+\left(\frac{2 \alpha-1}{2 \alpha}\right)^{n-2}+\ldots+\left(\frac{2 \alpha-1}{2 \alpha}\right)^{1 \text { or } 0}\right\} .
$$


Hence $k_{n}=O(1)$ if $\alpha>\frac{1}{4}$ and $\left|k_{n}\right| \sim 2 n$ if $\alpha=\frac{1}{4}$. Also, $Q_{n}=n+1$. All the conditions of theorem 19 are satisfied if $\alpha>\frac{1}{4}$. Hence for $\alpha>\frac{1}{4}, T\left(\alpha, \frac{1}{2}\right) \Rightarrow(C, 1)$, but there exists a sequence summable $T\left(\frac{1}{4}, \frac{1}{2}\right)$ which is not summable $(C, 1)$.

If $s=2$ and $\alpha=\frac{1}{4}$ then $k(x)=4\left(1-x^{2}\right)^{-2}$ and the conditions of theorem 19 are easily seen to hold, so that $T\left(\frac{1}{4}, \frac{1}{2}\right) \Rightarrow(C, 2)$.

(ii) The segment $\alpha>\frac{1}{4}$ of the line $2 \alpha+\beta=1$.

With $\left(N, p_{n}\right)$ and $\left(N, q_{n}\right)$ as the $T(\alpha, \beta)$ and $(C, s)$ processes respectively,

$$
\begin{aligned}
k(x) & =1 /\left\{\alpha(1-x)^{s}\left(1+\frac{1-2 \alpha}{\alpha} x+x^{2}\right)\right\} \\
& =\frac{1}{\alpha}(1-x)^{-s}(1-\gamma x)^{-1}\left(1-\frac{x}{\gamma}\right)^{-1} \\
& =(1-x)^{-s} \sum_{n=0}^{\infty}\left(a \gamma^{n}+b \gamma^{-n}\right) x^{n}
\end{aligned}
$$

where $\gamma=\{2 \alpha-1+i \sqrt{ }(4 \alpha-1)\} / 2 \alpha$ and $a, b$ are constants. If $s=1$, then

$$
k_{n}=\sum_{r=0}^{n}\left(a \gamma^{r}+b \gamma^{-r}\right)=O(1)
$$

since $\gamma \neq 1$ or -1 and $|\gamma|=1$. It follows that $T(\alpha, \beta) \Rightarrow(C, 1)$ when $\alpha>\frac{1}{4}$, $2 \alpha+\beta=1$.

\section{REFERENCES}

(1) R. P. Agnew, Inclusion relations among methods of summability, Arkiv for Matematile, 2, (1952) 361-374.

(2) G. H. Hardy, Divergent Series, Oxford, 1949.

(3) C. Hutton, Tracts Mathematical and Philosophical, London, 1812.

See also T. A. A. Broadbent : An early method for summation of series, Mathematical Gazette, 15 (1930), 5-11.

(4) W. Jurkat and A. Peyerimhoff, The consistency of Nörlund and Hausdorff methods, University of Cincinnatti Report No. 11 (1954).

(5) K. Knopp, Über das Eulersche Summierungsverfahren, Mathematische Zeitschrift, 15 (1922), 226-253.

(6) T. Kubota, Ein Satz über den Grenzwert, Tôholcu Mathematical Journal, 12 (1917), 222-224.

(7) L. L. Silverman and O. Szasz, On a class of Nörlund matrices, Annals of Mathematics, 45 (1944), 347-357.

Department of Mathematics UNIVERSTTY OF ST ANDREwS
Department of Mathematics UNIVERSITY OF THE WITSWATERSRAND 